\title{
Simulation numérique du comportement dynamique d'une transmission par engrenages en présence de défauts de dentures
}

\author{
Fakher ChaAri, Tahar Fakhfakh et Mohamed Haddar ${ }^{\mathrm{a}}$ \\ Unité de recherche Mécanique, Modelisation et Production, Département de Génie mécanique, École Nationale d'Ingénieurs \\ de Sfax, BP W, 3038 Sfax, Tunisie
}

Reçu le 15 janvier 2003, accepté le 2 juillet 2004

\begin{abstract}
Résumé - La modélisation d'une transmission par engrenages simple étage en mouvement permanent par un système à 2 degrés de liberté a permis de mettre en évidence un mode de corps rigide et un mode élastique. Le mode de corps rigide traduit la condition cinématique de roulement sans glissement et la condition de transmission de mouvement. Le mode élastique est la conséquence directe de l'élasticité des dents qui est à l'origine de l'erreur de transmission. La variation périodique de la raideur d'engrènement lors de la rotation constitue une source d'excitation interne pour la transmission. La détérioration d'une ou de plusieurs dents affecte la raideur d'engrènement et influe par conséquent sur le comportement dynamique de la transmission. Le but de cette étude est de simuler un type de défaut localisé sur une ou plusieurs dents ou bien réparti sur toutes les dents de façon à décrire approximativement un début de fissure, un écaillage, ... L'analyse de la réponse dynamique a permis alors de déterminer qualitativement l'influence de ce défaut. Des méthodes de détection appropriées au type de défaut sont utilisées. Il s'est avéré que pour les défauts localisés, la méthode du cepstre est très utile tandis que la méthode spectrale reste valable pour les défauts répartis.
\end{abstract}

Mots clés : Défauts d'engrenages / spectre / cepstre

\begin{abstract}
Numerical simulation of the dynamical behavior of a gear transmission set with tooth faults. The modeling of a one-stage spur gear transmission in permanent movement by a two-DOF system show us two modes: rigid body mode and elastic mode. Rigid body mode expresses the kinematics condition of rolling without slip and the state of transmission of movement. Elastic mode is the outcome of tooth elasticity, which is the origin of the static transmission error. The time varying Meshing stiffness is the main internal excitation source for the transmission. Deterioration of one or several teeth affects the gearmesh stiffness and consequently the dynamic behavior of the transmission. The purpose of this survey is to simulate a localized and distributed tooth faults in order to describe a beginning of crack, a scaling, ... Dynamic responses show that cepstrum analysis is very efficient for localized faults and spectrum analysis remains valid for distributed faults.
\end{abstract}

Key words: Gear faults / vibration analysis / spectrum / cepstrum

\section{Introduction}

Les transmissions par engrenages sont très répandues dans l'industrie. Elles sont d'une grande utilité lorsqu'il s'agit de transmettre des couples importants, de produire de grandes vitesses de rotation, d'effectuer un changement de direction du mouvement de rotation.

Les engrenages travaillent dans des conditions en général sévères et sont par conséquent soumis à une

a Auteur correspondant : mohamed_haddar@yahoo.fr détérioration progressive de leur état, notamment au niveau des dentures, d'où la nécessité de les soumettre à une surveillance conditionnelle continue afin de détecter à un stade précoce les éventuels défauts naissants [1].

Plusieurs méthodes de surveillance des machines ont été proposées dans la littérature. Manifestement, la plus répandue est l'analyse des vibrations [2-4]. En effet, cette méthode peut donner des renseignements qualitatifs sur les efforts développés au sein des organes de la machine et permet de détecter grâce à une technique appropriée les éventuels défauts. 
Récemment, plusieurs techniques de détection ont vu le jour [3]. Ces techniques sont plus ou moins adaptées selon le type de défaut. Ainsi, l'analyse spectrale peut détecter des défauts originaires de vibrations stationnaires et périodiques tels que les balourds. Cette technique reste néanmoins inefficace dès qu'il s'agit de détecter des défauts ayant un caractère non stationnaire [5] ou encore si plusieurs familles de périodicité sont présentes dans le signal.

La technique de détection par les ondelettes [6] a été utilisée pour le diagnostic des défauts localisés pouvant avoir indifféremment un caractère stationnaire ou non stationnaire.

La technique basée sur le cepstre a fait ses preuves quand il s'agit de détecter des défauts d'engrenages $[1,2,7,8]$. Les signaux représentatifs sont susceptibles de présenter plusieurs familles de périodicité. Cette technique permet de mettre en évidence la signature de défauts, modulant en amplitude ou en fréquence la réponse en l'absence de défauts.

On se propose dans ce travail d'analyser numériquement le comportement dynamique d'une transmission par engrenages simple étage, sans défauts de denture et en présence de défauts de denture localisés et répartis.

On développera dans un premier temps un modèle de réducteur à engrenages à 2 degrés de liberté en régime de fonctionnement permanent. Le modèle comprend deux modes de comportement dynamique. Le mode de corps rigide traduisant simplement une condition cinématique, exprime la condition de roulement sans glissement ainsi que l'égalité (au rapport $\eta$ de transmission près) entre les couples d'inertie moteur et récepteur. Le petit mouvement dû à la flexion des dents en prise, responsable de l'erreur de transmission, est décrit par le mode élastique.

Des résultats numériques concernant la réponse dynamique du mode élastique sont ensuite obtenus grâce à un algorithme d'intégration numérique en tenant compte de la contribution de l'engrènement parfait et de celle de l'engrènement présentant un défaut localisé, puis réparti.

La technique du cepstre a été utilisée et a permis de détecter certains types de défauts.

\section{Défauts d'engrenages}

On classe habituellement les défauts d'engrenages en trois catégories : les défauts de fabrication (erreurs de profil des dents, excentricité des roues, ...), les défauts de montage (défauts de parallélisme, d'entraxe, ...) et les défauts apparaissant lors du fonctionnement (usure ou fissuration des dents). Ces défauts expriment une erreur de transmission et entraînent une défaillance dans le fonctionnement du réducteur.

Les défauts de fabrication et de montage ont été étudiés par des récents travaux. Il a été démontré qu'une excentricité introduit une modulation d'amplitude - à la fréquence de rotation de la roue défectueuse - de la réponse marquée par la variation de la raideur d'engrènement [9]. Cela se traduit dans le domaine fréquentiel par l'apparition de raies latérales autour de la fréquence d'engrènement et de ses harmoniques. D'autres recherches expérimentales concernant les défauts de parallélisme et d'entraxes ont montré l'augmentation du niveau vibratoire de la machine, plus précisément l'accroissement dans la réponse des composantes qui correspondent à la fréquence de l'engrènement et ses harmoniques dans le domaine fréquentiel [10].

Le dysfonctionnement conséquent à l'usure des dents peut entraîner une perte de contact due à l'écart du profil idéal se traduisant par une diminution périodique de la raideur des dents. D'autre part, l'amorçage d'une fissure au pied d'une dent va provoquer une chute de raideur se produisant à la période de rotation de la roue defectueuse [11-13]. Ces deux types de défaut seront pris en compte dans le présent travail par une simulation numérique de la réponse dynamique d'un modèle de transmission par engrenages.

\section{Modélisation dynamique d'une transmission par engrenages droits simple étage}

On s'intéresse dans ce travail à la modélisation d'une transmission par engrenages simple étage à denture droite ne présentant pas de défauts de fabrication ou de montage. L'arbre moteur est soumis au couple moteur $T_{1}$, l'arbre récepteur est soumis au couple $T_{2}$ dû à la charge imposée à la sortie. Le mouvement de rotation de la transmission est supposé permanent et on se placera dans le cadre des petites perturbations autour de ce mouvement. Le rapport de transmission entre la roue et le pignon s'exprime par :

$$
\eta=\frac{r_{1}}{r_{2}}
$$

\subsection{Modélisation de la raideur d'engrènement}

Deux cas de figure peuvent être considérés, dans le premier la transmission est supposée infiniment rigide et dans le second on tiendra compte de sa flexibilité.

La transmission étant supposée infiniment rigide et la charge opposée par la machine réceptrice suffisante pour que le contact roue pignon soit assuré en permanence (pas de chocs), on ne devrait pas enregistrer de vibrations appréciables en l'absence de tout défaut et la transmission est dite idéale.

Dans la pratique, ceci n'est jamais le cas et de plus la transmission n'est pas infiniment rigide. En effet, quand l'arbre moteur est soumis à un couple de torsion, celui-ci est transmis à travers les dents de l'engrenage à l'arbre récepteur. Ces dernières opposent une raideur à la flexion et à l'écrasement. De même, le corps des roues ainsi que les arbres (moteur et récepteur) opposent une raideur à la torsion. Le couple statique est égal à une raideur équivalente $k_{\text {eq }}$ multipliée par la somme des déplacements 

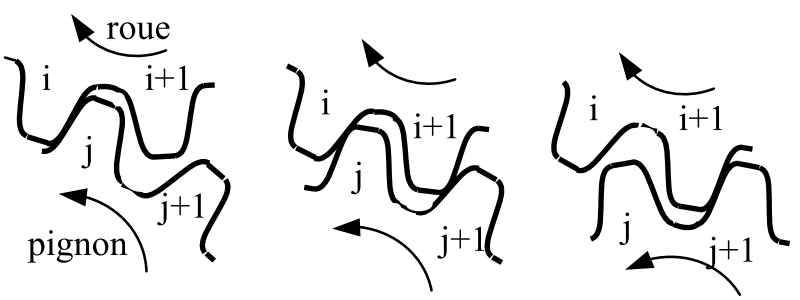

Fig. 1. Dents en prise lors de l'engrènement.

angulaires, qui représente l'erreur de transmission. Cette raideur équivalente est donnée par [14] :

$$
\frac{1}{k_{\mathrm{eq}}}=\frac{1}{k_{\mathrm{f}}}+\frac{1}{k_{\mathrm{ec}}}+\frac{1}{k_{\mathrm{v}}}+\frac{1}{k_{\mathrm{t}}}
$$

avec $k_{\mathrm{f}}$ la raideur de flexion des dents, $k_{\mathrm{ec}}$ la raideur à l'écrasement, $k_{\mathrm{v}}$ la raideur de voile du corps des roues et $k_{\mathrm{t}}$ la raideur à la torsion de la ligne d'arbres.

La raideur à l'écrasement des dents, la raideur de voile et la raideur à la torsion des arbres étant très grandes par rapport à la raideur à la flexion des dents, la raideur équivalente est donc pratiquement égale à cette dernière et est appelée raideur d'engrènement.

Par ailleurs, pendant la période d'engrènement $T_{\mathrm{e}}$ et pour un rapport de conduite $\varepsilon$ donné $(1<\varepsilon \leq 2)$, la roue et le pignon sont d'abord en contact par un seul couple de dents durant un laps de temps $(\varepsilon-1) T_{\mathrm{e}}$, puis par deux couples de dents durant $(2-\varepsilon) T_{\mathrm{e}}$.

La figure 1 présente la schématisation du phénomène d'engrènement.

Ainsi, la raideur d'engrènement est dans ce cas périodique de période $T_{\mathrm{e}}$ et peut être en première approximation estimée par [9] :

$$
k(t)=\left\{\begin{array}{lll}
k_{\max } & \text { si } \quad n T_{\mathrm{e}} \leq t \leq(n+\varepsilon-1) T_{\mathrm{e}} \\
k_{\min } & \text { si } \quad(n+\varepsilon-1) T_{\mathrm{e}} \leq t \leq(n+1) T_{\mathrm{e}}
\end{array}\right.
$$

$n$ entier.

Le développement en série de Fourrier de $k(t)$ donne :

$$
\begin{aligned}
k(t)=k_{\mathrm{m}}+\frac{\Delta k}{\pi} \sum_{i=1}^{\infty} \frac{1}{i} & {\left[\sin 2 i \pi(\varepsilon-1) \cos \frac{2 i \pi t}{T_{\mathrm{e}}}\right.} \\
& \left.+1-\cos 2 i \pi(\varepsilon-1) \sin \frac{2 i \pi t}{T_{\mathrm{e}}}\right]
\end{aligned}
$$

avec :

$$
\begin{aligned}
k_{m} & =k_{\max }(\varepsilon-1)+(2-\varepsilon) k_{\min } \\
\Delta k & =k_{\max }-k_{\min }
\end{aligned}
$$

$k_{\mathrm{m}}$ et $\Delta k$ sont positifs. On prendra $k_{\max }=6 \times 10^{7} \mathrm{~N} . \mathrm{m}^{-1}$, $k_{\text {min }}=3 \times 10^{7} \mathrm{~N} \cdot \mathrm{m}^{-1}$ et $\varepsilon=1,66$.

La figure $3 \mathrm{a}$ représente l'allure de $k(t)$ pendant quelques périodes d'engrènement.

\subsection{Modèle dynamique de la transmission}

Un train d'engrenages droits, simple étage peut être modélisé par un système à deux degrés de liberté $\theta_{1}(t)$

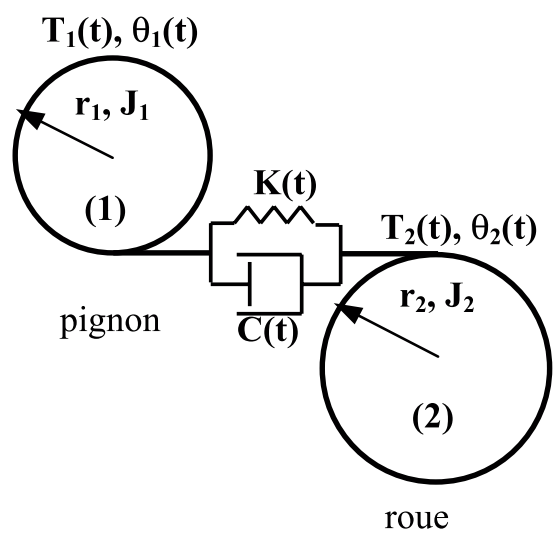

Fig. 2. Modèle dynamique de la transmission.
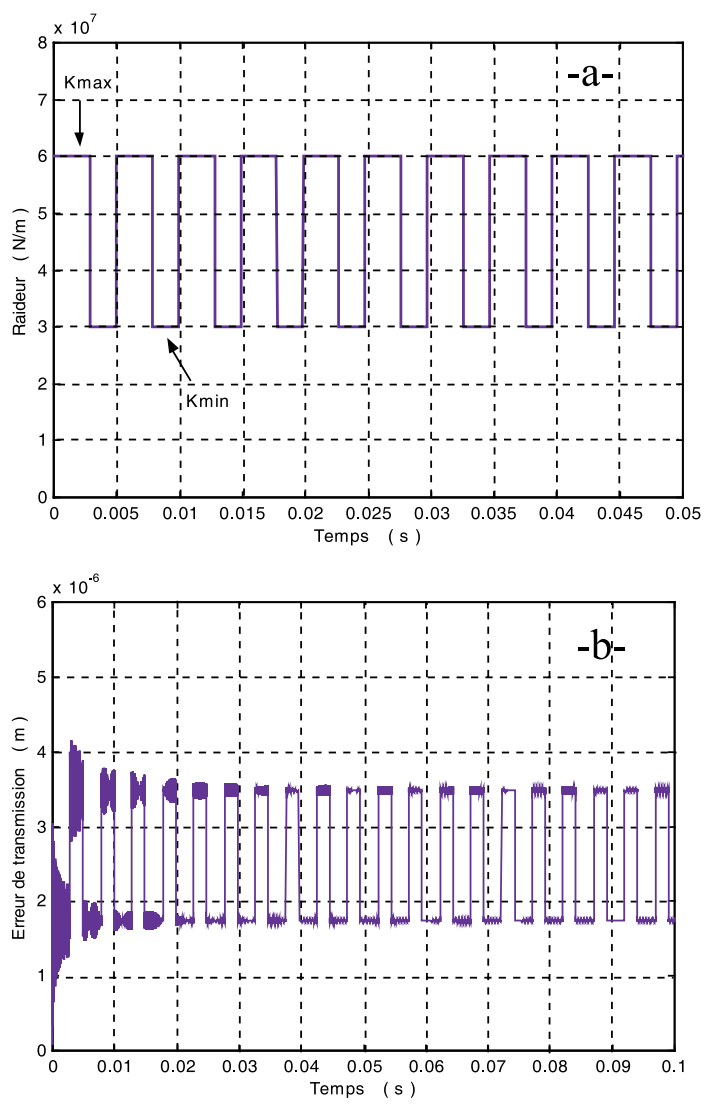

Fig. 3. Excitation et réponse temporelle de la transmission sans défauts de dentures.

et $\theta_{2}(t)[5,9,14]$, avec $\theta_{1}$ décrivant l'angle de rotation du pignon (1) et $\theta_{2}$ celui de la roue (2). Le pignon (1) a un rayon de base $r_{1}$, un moment d'inertie $J_{1}$ et est soumis au couple $T_{1}$. La roue (2) a un rayon de base $r_{2}$, un moment d'inertie $J_{2}$ et est soumise au couple $T_{2}$.

Pendant l'engrènement, les dents opposent une raideur à la flexion de coefficient $k(t)$ représenté par la figure $3 \mathrm{a}$ et un couple d'amortissement visqueux de coefficient d'amortissement $c(t)$ qui dépendent généralement du temps selon qu'un seul ou bien deux couples de dents sont engagés lors de la transmission du mouvement (Fig. 1). 
L'écriture des énergies cinétique et potentielle du modèle mène, compte tenu du formalisme de Lagrange, aux équations de mouvement :

$$
M \ddot{\vec{\theta}}(t)+C(t) \dot{\vec{\theta}}(t)+K(t) \vec{\theta}(t)=\vec{F}(t)
$$

avec :

$$
\begin{gathered}
M=\left(\begin{array}{ll}
J_{1} & 0 \\
0 & J_{2}
\end{array}\right), C(t)=c(t)\left(\begin{array}{cc}
r_{1}^{2} & r_{1} r_{2} \\
r_{1} r_{2} & r_{2}^{2}
\end{array}\right) \\
K(t)=k(t)\left(\begin{array}{cc}
r_{1}^{2} & r_{1} r_{2} \\
r_{1} r_{2} & r_{2}^{2}
\end{array}\right), \vec{\theta}(t)=\left(\begin{array}{cc}
\theta_{1}(t) \\
\theta_{2}(t)
\end{array}\right)
\end{gathered}
$$

et

$$
\vec{F}(t)=\left(\begin{array}{l}
T_{1}(t) \\
T_{2}(t)
\end{array}\right)
$$

respectivement les matrices d'inertie d'amortissement et de raideur et les vecteurs déplacements angulaires et couples extérieurs appliqués à la roue et au pignon.

La fonction $k(t)$ étant donnée par (3), l'équation (7) est de la forme :

$$
M \ddot{\vec{\theta}}(t)+\left[b^{2}+\beta(t)\right] \dot{\vec{\theta}}(t)+\left[a^{2}+\alpha(t)\right] \vec{\theta}(t)=\vec{F}(t)
$$

qui est une équation de Hill [1] et l'on remarquera que pour les données du problème, la solution de (8) est stable.

Une base modale du système libre non amorti de matrice de raideur moyenne :

$$
\tilde{K}(t)=k_{\mathrm{m}}\left(\begin{array}{cc}
r_{1}^{2} & r_{1} r_{2} \\
r_{1} r_{2} & r_{2}^{2}
\end{array}\right)
$$

peut facilement être obtenue. On trouve un mode de « corps rigide » de vecteur $\alpha\left(r_{2}-r_{1}\right)^{T}$ et un mode « élastique » de pulsation propre :

$$
\omega_{0}^{2}=k_{\mathrm{m}} \frac{\left(J_{1} r_{2}^{2}+J_{2} r_{1}^{2}\right)}{J_{1} J_{2}}
$$

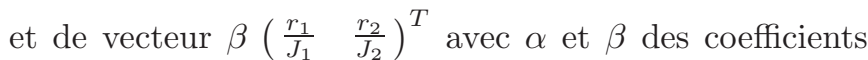
de proportionnalité. On prendra pour des raisons de commodité :

$$
\left\{\begin{array}{l}
\alpha=1 \\
\beta=m_{\mathrm{e}}=\frac{J_{1} J_{2}}{J_{1} r_{2}^{2}+J_{2} r_{1}^{2}}
\end{array}\right.
$$

$m_{\mathrm{e}}$ une masse équivalente. La matrice de changement de coordonnées :

$$
\Phi=\left(\begin{array}{lll}
r_{2} & m_{\mathrm{e}} \frac{r_{1}}{J_{1}} \\
-r_{1} & m_{\mathrm{e}} \frac{r_{2}}{J_{2}}
\end{array}\right)
$$

exprime les coordonnées $\left(\begin{array}{ll}\theta_{1} & \theta_{2}\end{array}\right)^{T}$ en fonctions des coordonnées généralisées $\left(\begin{array}{ll}q_{1} & q_{2}\end{array}\right)^{T}$. La projection sur cette base modale de vecteurs propres orthogonaux permet d'écrire - à partir de (7) - les équations de mouvement :

$$
\left\{\begin{array}{l}
\frac{J_{1}}{r_{1}} \frac{J_{2}}{r_{2}} \ddot{q}_{1}(t)=m_{\mathrm{e}}\left(\frac{T_{1}}{r_{1}}-\frac{T_{2}}{r_{2}}\right) \\
m_{\mathrm{e}} \ddot{q}_{2}(t)+c(t) \dot{q}_{2}(t)+k(t) q_{2}(t)= \\
m_{\mathrm{e}}\left(\frac{r_{1}}{J_{1}} T_{1}+\frac{r_{2}}{J_{2}} T_{2}\right)
\end{array}\right.
$$

avec :

$$
\left\{\begin{array}{l}
q_{1}(t)=m_{\mathrm{e}}\left(\frac{r_{2}}{J_{2}} \theta_{1}(t)-\frac{r_{1}}{J_{1}} \theta_{2}(t)\right) \\
q_{2}(t)=r_{1} \theta_{1}(t)+r_{2} \theta_{2}(t)
\end{array}\right.
$$

Pour des roues de 22 et 44 dents, de module $m=2$ et d'épaisseur 0,02 $\mathrm{m}$, en acier de masse volumique $\rho=$ $7800 \mathrm{~kg} . \mathrm{m}^{-3}$, la fréquence propre moyenne $f_{0}$ de la transmission est de l'ordre de $4 \times 10^{4} \mathrm{~Hz}$, très supérieure à $f_{\mathrm{e}}$.

La première équation de (13) représente le mouvement de corps rigide. Lors du mouvement de rotation permanent de la transmission, le second membre de cette équation est nul et l'on obtient (au coefficient de réduction près) l'égalité entre les vitesses de rotation de la roue et du pignon ainsi que l'égalité entre les couples d'inertie moteur et récepteur.

La seconde équation représente le mode élastique. La coordonnée généralisée $q_{2}(t)$ qui décrit le mouvement élastique est l'erreur de transmission. C'est la source d'excitation de la boîte de transmission [15].

L'intégration numérique de cette équation pour une variation donnée de la raideur d'engrènement permettrait de comprendre le comportement dynamique de la transmission par engrenages.

\section{Simulation numérique}

L'équation du mouvement représentative du mode élastique seconde équation du système (13) est une équation différentielle du second ordre à coefficients variables. La résolution est effectuée par la méthode d'intégration de Newmark [17].

Dans un premier temps, la paire de roues est supposée parfaite. Ensuite, une modélisation d'un défaut réparti puis un défaut localisé sur le pignon ou sur la roue ont été considérés.

La réponse est présentée dans les domaines temporel et fréquentiel. La technique du cepstre a été utilisée pour la visualisation du caractère dû au défaut (voir annexe).

\subsection{Dentures saines}

Dans ce cas, la variation de la raideur est donnée par (3) et est illustrée par la figure 3a.

La réponse $q_{2}(t)$ du modèle est représentée par la figure $3 \mathrm{~b}$, et l'on vérifie bien son caractère stable. 

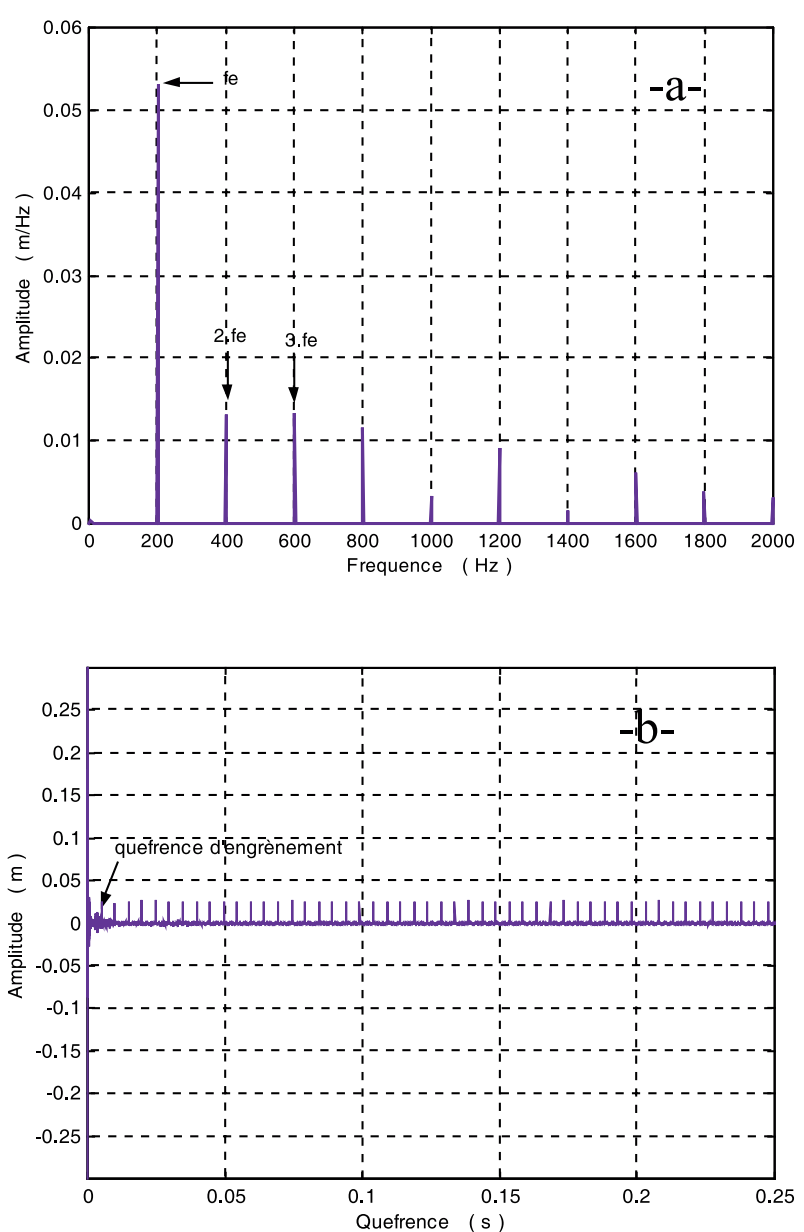

Fig. 4. Spectre et cepstre de la réponse pour la transmission sans défauts de dentures.

Ceci justifie d'écrire que la réponse est pratiquement périodique de pulsation fondamentale $\omega_{\mathrm{e}}[5,6]$ :

$$
q_{2}(t)=\sum_{m=0}^{M} \gamma_{\mathrm{m}} \cos \left(m \omega_{\mathrm{e}} t+\varphi_{\mathrm{m}}\right)
$$

$M$ entier grand.

La figure 4a représente le module du spectre de la réponse dans la bande de fréquence [0-2000 Hz]. Ce dernier est marqué par la fréquence d'engrènement et ses harmoniques. La figure $4 \mathrm{~b}$ représente le cepstre de la réponse sur laquelle on constate la prédominance de la quefrence d'engrènement et ses rahmoniques.

\subsection{Défaut de dentures}

\subsubsection{Défauts localisés}

Un exemple d'un tel défaut est représenté par la figure 5a. On constate que l'expression de $k(t)$ est périodique de pulsation $\omega_{\mathrm{r}}=2 \pi / T_{r}(r=1$ ou 2 selon que le défaut est sur le pignon ou sur la roue).

L'amorçage d'un défaut sur une dent va donc se traduire par des «impulsions » dans la réponse temporelle
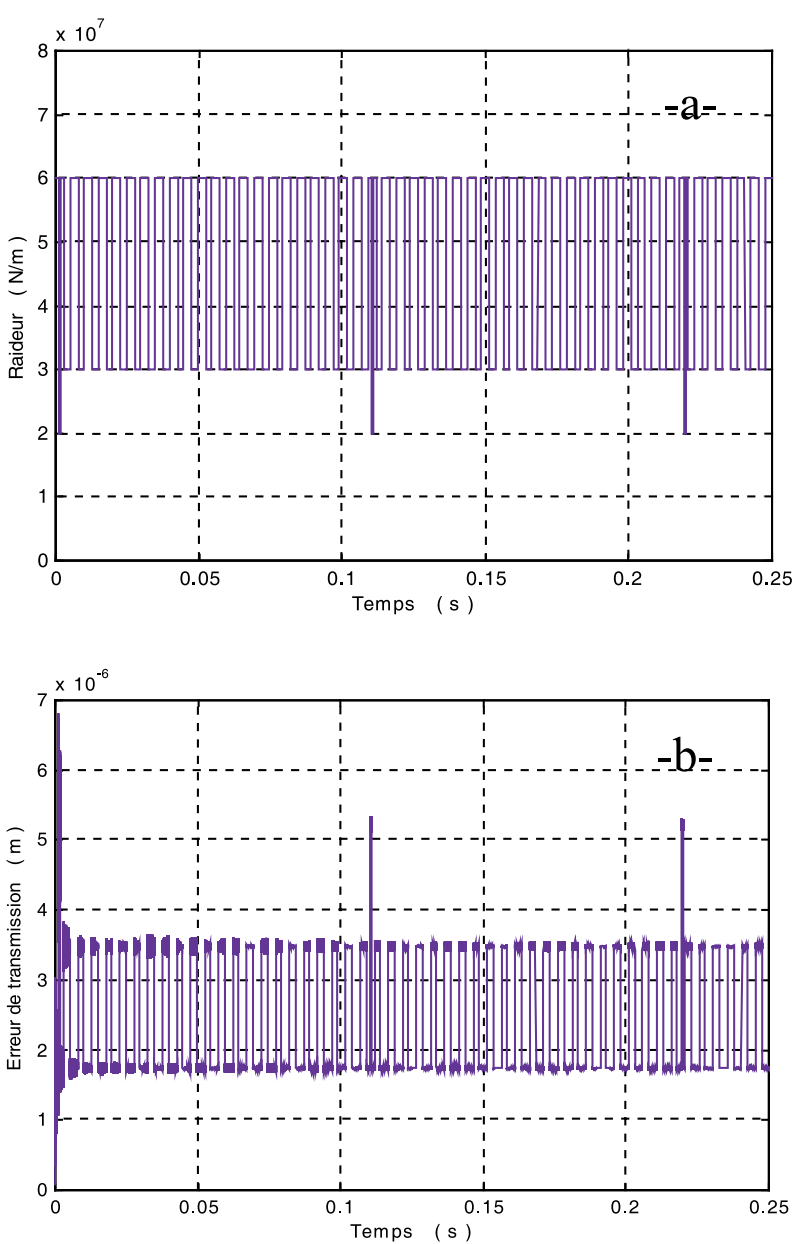

Fig. 5. Raideur d'engrènement avec défaut localisé sur le pignon et réponse temporelle $\left(N_{1}=550 \mathrm{tr} \cdot \mathrm{min}^{-1}, \eta=0,5\right)$.

qui vont se répéter à chaque engrènement de cette dent (Fig. 5b).

Le signal associé à ce défaut va moduler en amplitude le signal dû à l'engrènement et on aura un spectre plus complexe, caractérisé par l'apparition de bandes latérales autour de la fréquence d'engrènement et de ses harmoniques (Fig. 6a). De façon générale, la fonction modulante s'écrit [1] :

$$
a(t)=\sum_{m=0}^{M} a_{\mathrm{m}} \cos \left(m \omega_{\mathrm{r}} t+\alpha_{\mathrm{m}}\right)
$$

La réponse $q_{2}(t)$ du modèle - compte tenu de son caractère stable - va s'écrire sous la forme $[5,6]$ :

$$
q_{2}(t)=\sum_{m=0}^{M} \gamma_{\mathrm{m}}(1+a(t)) \cos \left(m \omega_{\mathrm{e}} t+\varphi_{\mathrm{m}}\right)
$$

On présente sur les figures $6 \mathrm{a}$ et $6 \mathrm{~b}$ le module du spectre et le cepstre de la réponse après intégration numérique de (13) compte tenu du défaut localisé sur le pignon. 

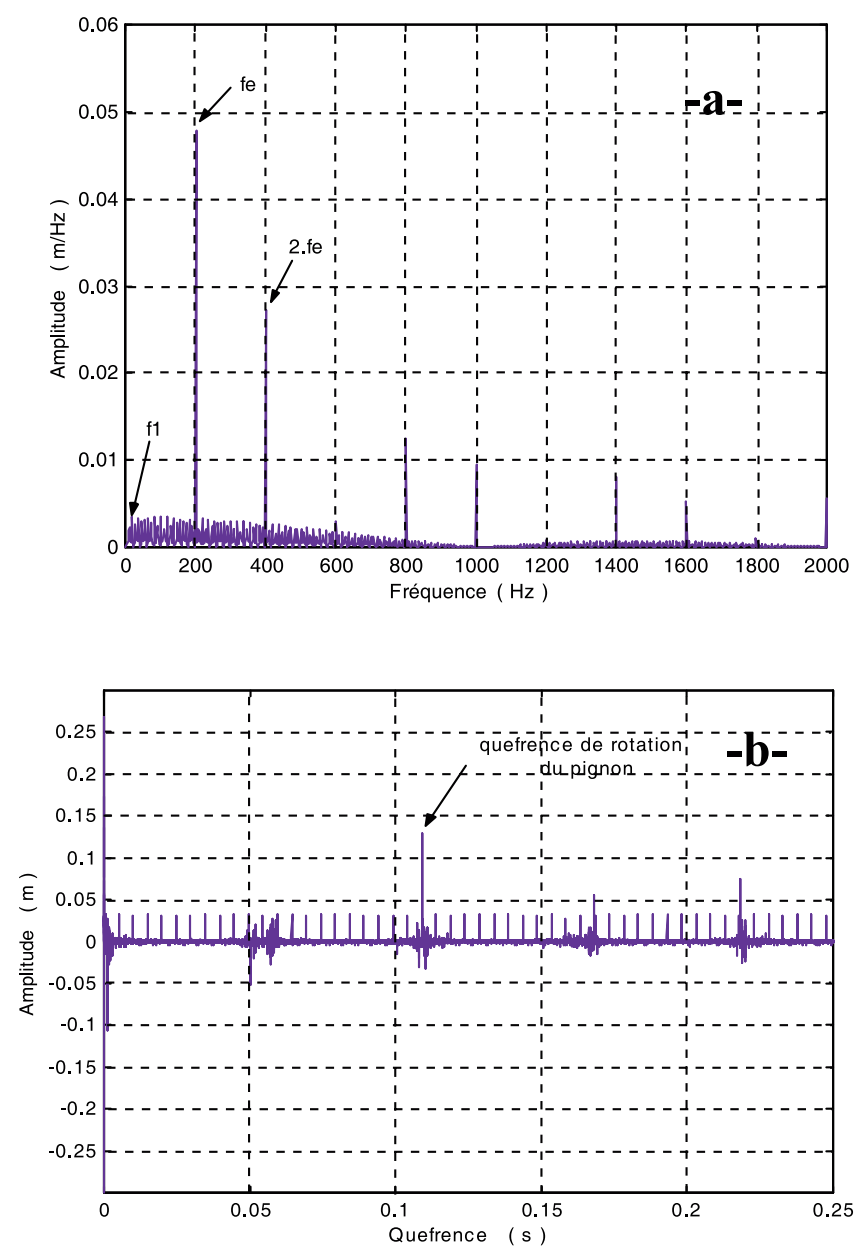

Fig. 6. Spectre et cepstre de la réponse due à un défaut localisé sur le pignon.

Le cepstre (voir annexe) permet de dissocier l'effet de la fonction modulante du signal d'engrènement et met en évidence la présence du défaut.

Par rapport au cepstre de la réponse de la transmission sans défauts, on constate l'augmentation de la quefrence de rotation du pignon et les rahmoniques correspondants.

Quand le défaut concerne de plus en plus de dentures, l'activité des raies latérales les plus proches de $f_{\mathrm{e}}$ et de ses harmoniques gagne de l'importance sur celle des raies éloignées. Le spectre prend la forme de paquets de raies (Fig. 7a). Ceci vient du fait que la fonction modulante (qui caractérise le défaut) est de plus en plus régulière [8]. Sur le cepstre, on constate l'augmentation de l'activité de la quefrence d'engrènement et des rahmoniques correspondants.

\subsubsection{Défauts répartis}

Dans le cas de défauts répartis, l'expression de $k(t)$ est périodique de pulsation fondamentale $\omega_{\mathrm{e}}$ (Fig. 8a).
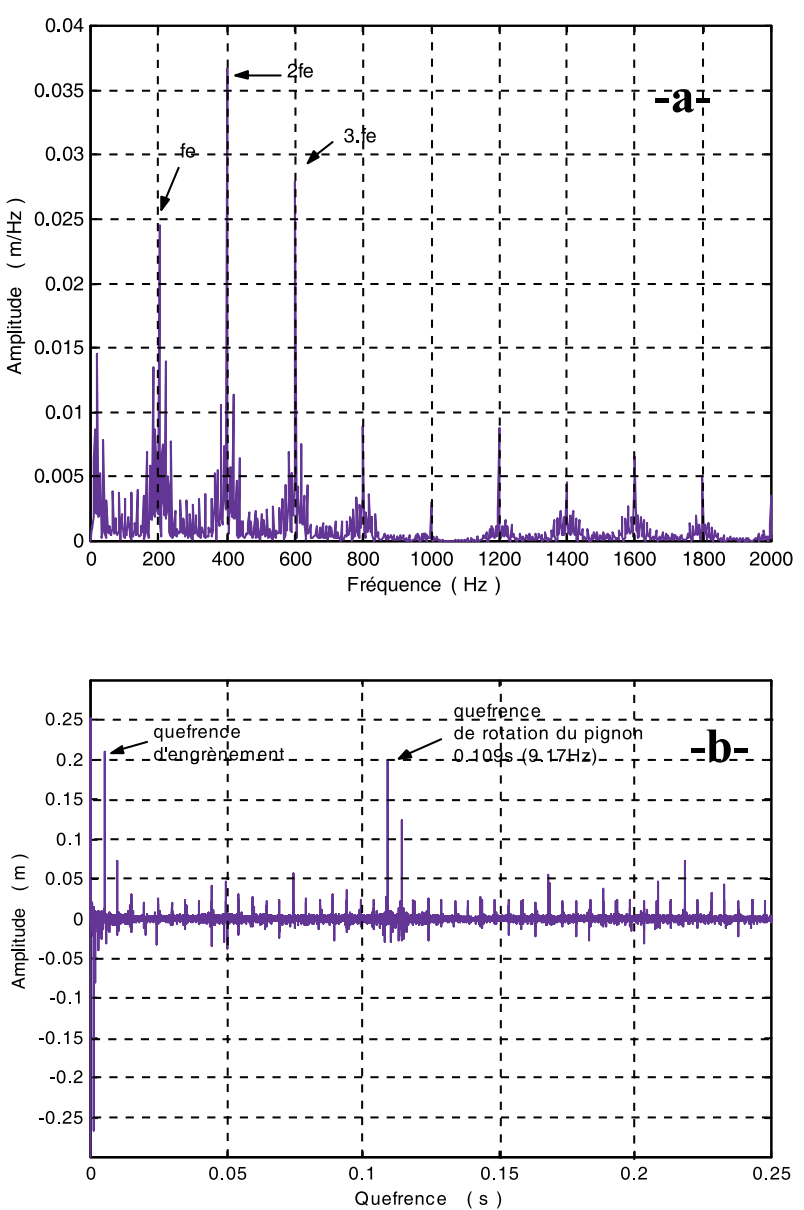

Fig. 7. Spectre et cepstre de la réponse due à des défauts répartis sur quelques dents du pignon.

La réponse $q_{2}(t)$ peut dans ce cas s'écrire $[5,6]$ :

$$
q_{2}(t)=\sum_{m=0}^{M} \gamma_{\mathrm{m}}(1+b(t)) \cos \left(m \omega_{\mathrm{e}} t+\varphi_{\mathrm{m}}\right)
$$

avec :

$$
b(t)=\sum_{m=0}^{M} b_{\mathrm{m}} \cos \left(m \omega_{\mathrm{e}} t+\alpha_{\mathrm{m}}\right)
$$

$b(t)$ étant le signal du défaut modulant en amplitude celui de l'engrènement.

Le défaut réparti sur toutes les dents se manifeste sur le spectre par une augmentation de l'amplitude de la fréquence d'engrènement et ses harmoniques (Fig. 9a). Cependant le cepstre est quasi indifférent pour ce défaut.

\section{Conclusion}

La transmission par engrenages droits simple étage a été modélisée par un système à deux degrés de liberté à raideur variable dans le temps (fonction des paires de dents en prise). 

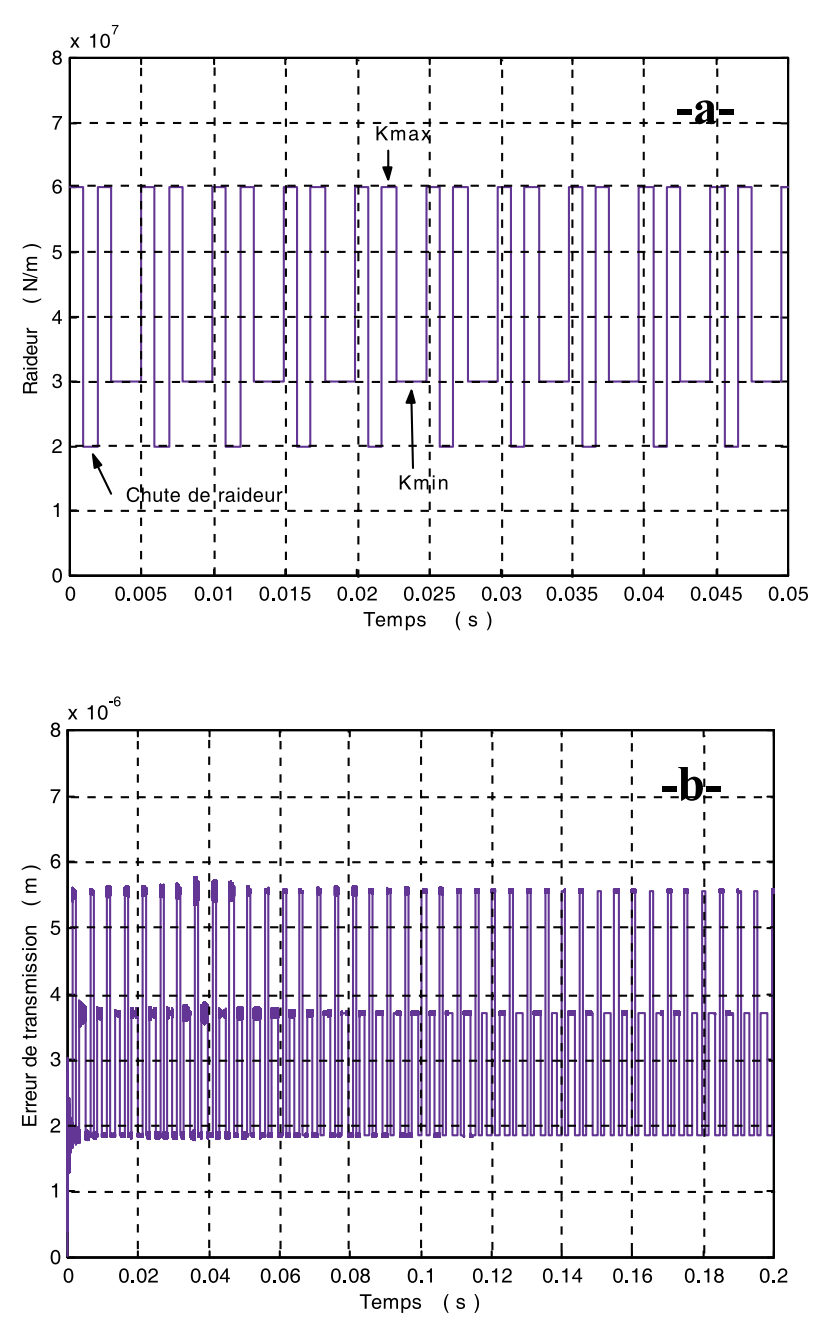

Fig. 8. Raideur d'engrènement et réponse temporelle avec défauts répartis identiquement sur toutes les dents du pignon $\left(N_{1}=550\right.$ tr. $\left.\min ^{-1}, \eta=0,5\right)$.

Le modèle adopté présente un mode de corps rigide et un mode élastique. Il a permis d'étudier l'effet de l'erreur de transmission dû au phénomène d'engrènement sur le comportement dynamique du système de transmission par engrenages. L'erreur de transmission est décrite par le mode élastique du modèle. Le comportement dynamique du système de transmission est marqué par la fréquence d'engrènement et ses harmoniques.

L'analyse de Fourier de la réponse du modèle confirme ce résultat.

Un défaut d'engrenages survenant au cours du fonctionnement affecte essentiellement les dents de la roue ou du pignon. Il peut être localisé ou réparti. Il se manifeste par une chute périodique de la raideur de l'engrènement, de période - selon le type du défaut - celle de la rotation de la roue comportant le défaut ou bien celle de l'engrènement. La réponse du modèle dynamique à de tels défauts a été étudiée. Cette réponse est caractérisée par une modulation d'amplitude des composantes de la fréquence d'engrènement et de ses harmoniques par la périodicité du défaut.
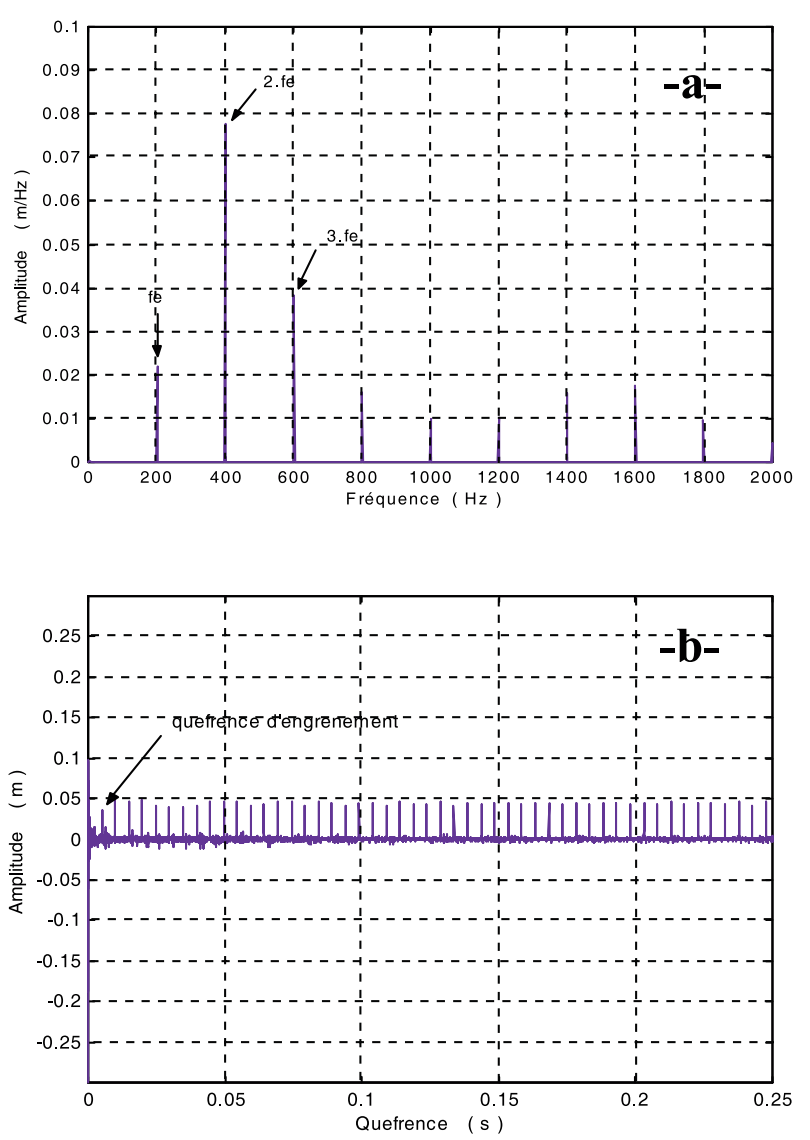

Fig. 9. Spectre et cepstre de la réponse due à un défaut réparti sur toutes les dents du pignon.

Dans le cas de défauts localisés, l'analyse de Fourier a permis de mettre en évidence l'aspect de modulation d'amplitude par l'apparition de raies latérales dans le spectre de la réponse aux fréquences de rotation, notamment à la fréquence de rotation de la roue comportant le défaut.

L'analyse cepstrale a l'avantage d'extraire cette fréquence qui traduit le défaut. Elle est d'autant plus utile que les vitesses de rotation sont basses et que le nombre d'étages de la transmission multiple, car dans ce cas, le paquet de raies latérales présent dans le spectre de la réponse devient très compact et difficile à discerner surtout en présence d'autres composantes spectrales provenant de machines adjacentes.

Dans le cas de défauts répartis, l'analyse de Fourier semble être suffisante pour détecter ce type de défauts. Ces derniers se manifestent par une augmentation de l'amplitude de la réponse, c'est-à-dire aussi de celles de ses composantes spectrales.

\section{ANNEXE : Technique du cepstre}

La modulation d'amplitude d'un signal périodique de fréquence $f_{\mathrm{e}}$ élevée (par exemple la fréquence d'engrènement) par un ou plusieurs autres signaux périodiques de fréquences $f_{\text {ri }}$ (par exemple le signal qui 

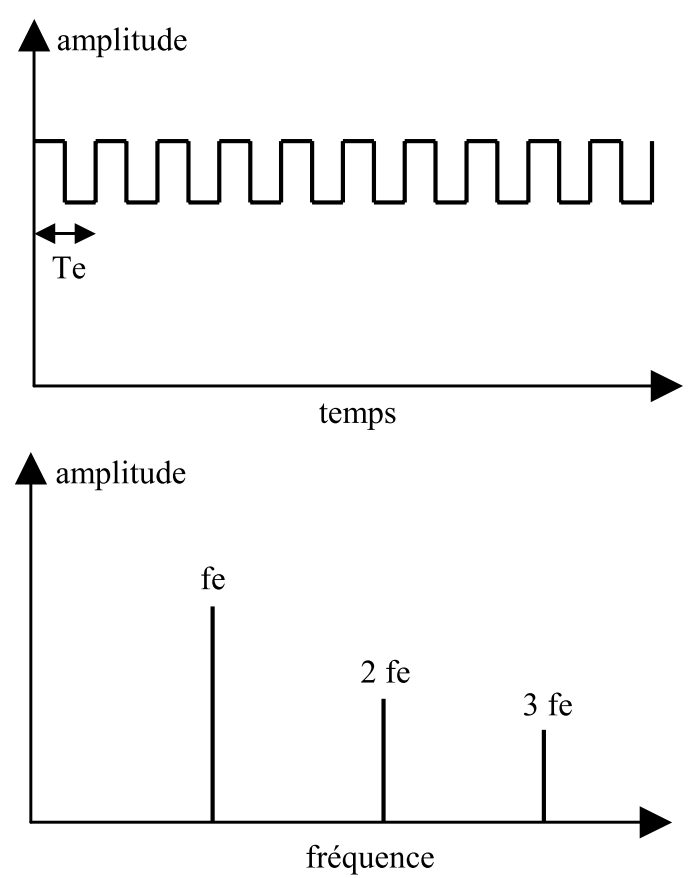

Fig. A.1. Réponse temporelle et fréquentielle du signal sans défaut.

représente le défaut présent au niveau de la roue ou du pignon) se caractérise dans le domaine fréquentiel par l'apparition de raies latérales autour de la fréquence fe (c'est-à-dire de fréquence $f_{\mathrm{e}} \pm m f_{\mathrm{ri}}$ ) et de ses harmoniques (c'est-à-dire de fréquences $n f_{\mathrm{e}} \pm m f_{\text {ri }}[1]$ ).

Quand l'espacement entre raies devient plus étroit, l'analyse spectrale devient difficile et la distinction entre les différentes périodicités sera moins aisée. Le cepstre est en mesure de résoudre ce problème.

Il est défini comme étant « la transformée de Fourier inverse du logarithme du spectre de puissance $»[2,7,8]$ :

$$
\mathrm{Ce}(\tau)=T F^{-1}\{\log S(\nu)\}
$$

Il permet, tout d'abord, en passant au logarithme de dissocier les différents signaux modulant et d'amplifier les composantes de faible amplitude. Ensuite, en effectuant la transformée de Fourier inverse, il fournit des peignes de raies correspondant à chacun des organes tournant au pas de chacune des périodes de rotation.

Le suivi de l'évolution de l'amplitude des différents peignes fournit un outil de diagnostic de première importance. Ainsi, on a pu démontrer [7] que pour un système à $M$ engrenages donc à $M$ peignes de raies aux pas de chacune des périodes de rotation, la somme des premiers pics de chaque peigne est constante. Par conséquent, lorsqu'un défaut se développe sur un engrenage, l'amplitude du peigne correspondant va augmenter au détriment des autres. Cette méthode est d'autant plus efficace que la fréquence du défaut est faible (défaut localisé). Sur la figure A.1 on présente la réponse temporelle d'un système : elle est périodique de période $T_{\mathrm{e}}$. Ce système va être excité par un signal de défaut présentant des impulsions périodiques se produisant à la période $T_{\mathrm{r}}$ (Fig. A.2).
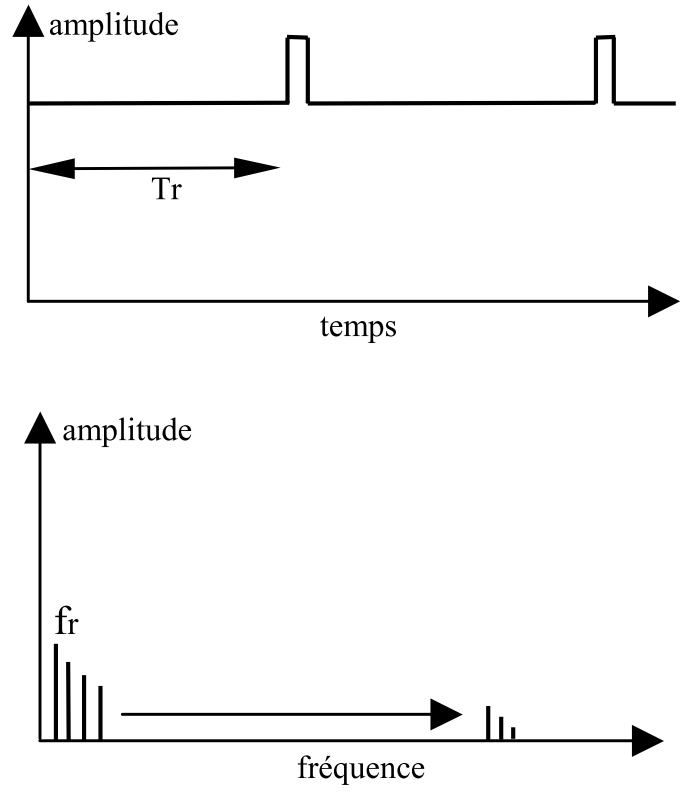

Fig. A.2. Signal d'enveloppe dû au défaut et spectre correspondant.
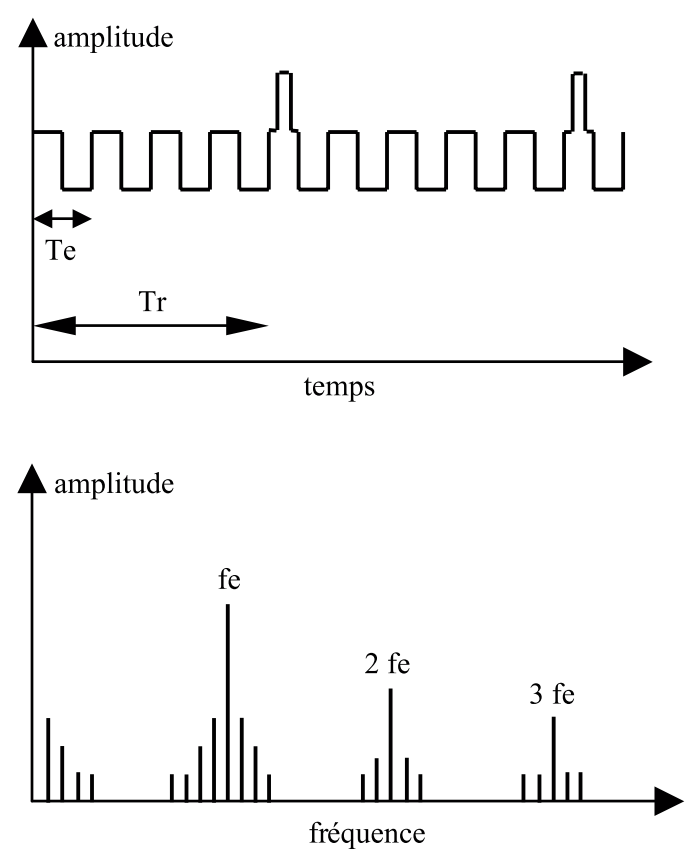

Fig. A.3. Modulation d'amplitude de $f_{\mathrm{e}}$ par $f_{\mathrm{r}}$.

Ce signal va moduler en amplitude le signal initial. Le résultat dans le domaine fréquentiel est obtenu en effectuant le produit de convolution des deux signaux (Fig. A.3). Le passage au cepstre (Fig. A.4) permet de mettre en évidence le défaut par l'augmentation du peigne y afférent. 


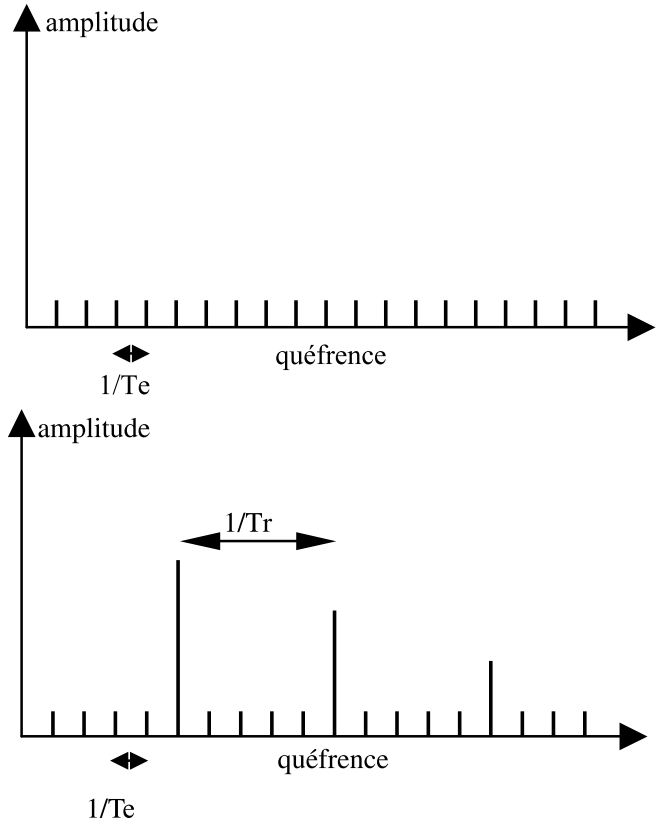

Fig. A.4. Cepstre avant et après modulation.

\section{Références}

[1] R. Bigret, J.L. Freon, Diagnostic, maintenance, disponibilité des machines tournantes, Édition Masson, Paris, France, 1995

[2] C. Capdessus, M. Sidahmed, Analyse des vibrations d'un engrenage : cepstre, corrélation, spectre, traitement du signal, 1991, 8, $\mathrm{N}^{\circ} 5$

[3] M. Lebold, K. McClintic, R. Cambell, C. Byington, K. Maynard, Review of vibration analysis methods for gearbox diagnostics and prognostics, Proceedings of the 54th Meeting of the Society for Machinery Failure Prevention Technology, Virginia Beach, VA, May 1-4, 2000

[4] M. Sidahmed, Détection précoce des défauts d'engrenages par analyse vibratoire, $4^{\mathrm{e}}$ journées d'études transmissions mécaniques, ECAM Lyon, juin 1990

[5] L. Bouillaut, Approches cyclostationnaire et bilinéaire pour l'analyse vibratoire de machines tournantes : Approches théoriques et applications au diagnostic, Thèse de l'Université de technologie de Compiègne, 2000
[6] A.J. Miller, A new wavelet basis for the decomposition of gear motion error signals and its application to gearbox diagnostics, Thèse de l'université de l'état de Pennsylvanie, 1999

[7] M. El Badaoui, F. Guillet, J. Daniere, Surveillance des systèmes complexes à engrenages par l'analyse cepstrale synchrone, Traitement du signal 16 (1999), 5

[8] R.B. Randall, Cepstrum analysis and gearbox fault diagnosis, Edition 1, Bruel and Kjaer, Application note, 1977

[9] M. Merzoug, A. Miloudi, K. Brahimi, Comportement dynamique d'une transmission par engrenages - Analyse de la stabilité, $\mathrm{XV}^{\mathrm{e}}$ Congrès Français de Mécanique, Nancy, 3-7 septembre 2001

[10] M. Haddar, M. Ben Amar, W. Gafsi, A. Maalej, Analyse du comportement dynamique et tribologique d'une transmission à engrenages droits en présence d'un défaut de parallélisme, $3^{\mathrm{e}}$ journées de mécanique et ingénierie, Sfax, 28-30 mars 2000

[11] C.D. Begg, C.S. Byington, K.P. Maynard, Dynamic Simulation of Mechanical Fault Transition, Proceedings of the 54th Meeting of the Society for Machinery Failure Prevention Technology, Virginia Beach, VA, May 1-4, 2000

[12] G. Dalpiaz, A. Rivola, R. Rubini, Dynamic modeling of gear systems for condition monitoring and diagnostics, Congress on Technical Diagnostics, 1996

[13] M. Haddar, Réponse dynamique d'une boîte de vitesse, Revue internationale d'ingénierie des systèmes de productions mécaniques (1998), V-29-V-34

[14] P. Velex, Contribution à l'analyse du comportement dynamique des engrenages à axes parallèles, Thèse de doctorat : Inst. Nat. des sciences appliquées de Lyon, 1988

[15] E. Rigaud, Modeling and analysis of static transmission error-Effect of wheel deformation and interactions between adjacent loaded teeth, Laboratoire de tribologie et dynamique des systèmes, École centrale de Lyon

[16] K.P. Maynard, Interstitial processing : the application of noise processing gear fault detection, Proceedings of the international conference on Condition monitoring, University of Wales Swansea, UK, 12th April 1999, pp. $77-86$

[17] G. Dhatt, G. Touzot, Une présentation de la méthode des éléments finis, Édition Maloine, Paris, France, 1984

Retrouvez nos articles sur le site : www.edpsciences.org/meca 